\title{
Is Early Detection of Colon Cancer Possible with Red Blood Cell Distribution Width?
}

\author{
Serden Ay ${ }^{1 *}$, Mehmet Ali Eryilmaz ${ }^{2}$, Nergis Aksoy², Ahmet Okus ${ }^{3}$, Yasar Unlu ${ }^{4}$, \\ Barıs Sevinc $^{2}$
}

\begin{abstract}
Background: Red cell distribution width (RDW) is one of the standard parameters with blood cell counts. Much previous research has indicated that it increases in cases of systemic inflammation or cardiametabolic incident. However, information on the relation of RDW with solid tumors causing systemic inflammation is limited. In the present research, we examined the relation of RDW with malignant and benign lesions of the colon. Materials and Methods: 115 patients with colon polyps (group 1), and 30 with colon cancer (group 2) who were diagnosed histopathologically in our clinic between January 2010-January 2013 were scanned retrospectively. Patients with anemia, hematologic diseases and active inflammation were excluded. RDW, mean corpuscular volume (MCV), hemoglobin (Hgb) and platelet (PIt) measurements were recorded and their relations with the malignant and benign lesions of the colon were examined. Results: Both groups were similar in age and gender distribution. RDW values of patients with colon cancer were significantly higher than the patients with colon polyp $(p=0,01)$. No significant differences were detected between the two groups in terms of MCV and PIt values $(p>0,05)$. Conclusions: RDW can be used as an early warning biomarker for solid colon tumors. Further prospective research is required on the relations of cheap and easily measured RDW parameters with colon malignancies.
\end{abstract}

Keywords: Colorectal cancer - colon polyp - red cell distribution width - associations

Asian Pac J Cancer Prev, 16 (2), 753-756

\section{Introduction}

Red blood cell distribution width (RDW) is one of the parameters in the standard complete blood count and it reflects the heterogeneity of the sizes of the red blood cells in blood circulation. The clinical use of RDW was previously limited to distinguishing iron deficiency anaemia from thalassaemia and other haemoglobinopathies among the anaemias which lead to microcytic anaemia. However, recent studies have reported that RDW increased in atherosclerosis, ischemic cardiac diseases, acute and chronic cardiac failure, hypertension, the detection of the activity of inflammatory intestinal diseases, prostate cancer and generally increased progressive inflammations (Yesil et al., 2011; Gunebakmaz et al., 2012; Karabulut and Uzunlar, 2012; Nishizaki et al., 2012; Albayrak et al., 2014). It is reported that inflammation and oxidative stress influenced RDW (Patel et al., 2009). Furthermore, it was shown that RDW also reflected the increase in the cytokines such as IL6, TNF-a and hepcidin circulating in blood (Rhodes et al., 2011; de Gonzalo et al., 2012). Cancer, on the other hand is characterized with increased inflammation and RDW was rarely examined in solid tumours (Speights et al., 1992; Ozkalemkas et al., 2005; Spell et al., 2004; Baicus et al., 2011; Beyazit et al., 2012; Seretis et al., 2013). And we, in our study, researched if RDW can be a biomarker which can be used in the early detection of colon cancer.

\section{Materials and Methods}

The files of 110 colon polyp (group 1) and 30 colon cancer (group 2) patients which were diagnosed upon the histopathological examination of the biopsy materials which were taken from the lesions detected during the colonoscopy which was made in endoscopy unit of Konya Education and Research Hospital General Surgery clinic between January 2010- January 2013 were retrospectively scanned. From the complete blood counts of the patients within maximum one week before being diagnosed, the mean cell volume (MCV), haemoglobin (hgb), platelet (plt) and RDW values were recorded. The patients with anaemia, haematological disorder, active infection, with blood transfusion made in the last three months, venous thrombosis detected in the last six months, receiving iron deficiency treatment with their histories scanned and the 
patients with hypertension, cardiac failure, inflammatory intestinal disease and rheumatoid arthritis whose RDW values seen to have increased in the previous studies were extracted from the study because their RDW values would be affected. We compared the patients in whom colon cancer was detected in the colon during colonoscopy with the patients in whom only polypoid lesion was detected in the colon in terms of haematological values mainly including RDW.

SPSS (Statistical Package for Social Sciences) for Windows 18.0 programme was used for statistical analysis. All parameters are normally distributed and presented with mean and \pm S.D. Categorical variables are shown as frequencies. Continuous variables were correlated using independent samples t-test, while chisquare was used when comparing categorical variables. A P $<0.05$ level was considered statistically significant. RDW values in predicting colon cancer were analyzed using Receiver Operating Characteristics (ROC) curve analysis. When a significant cut-off value was observed, the sensitivity, specificity were presented.

\section{Results}

The age average of group 1 was $60,2 \pm 5,8$ while the

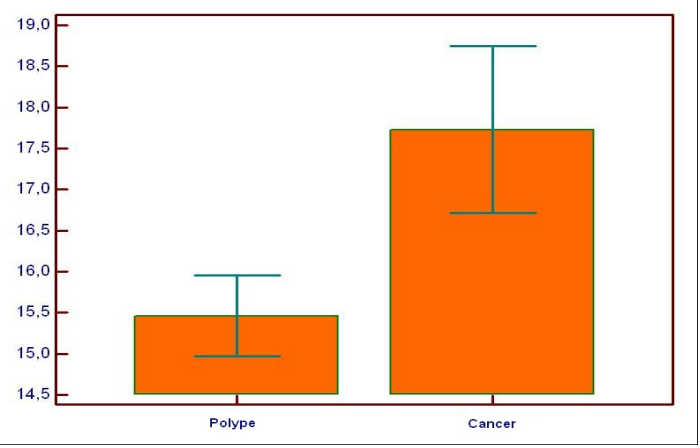

Figure 1. Comparison of Groups in Terms of RDW values

Table 1. Laboratory Parameters of Study Population

\begin{tabular}{lccc}
\hline Parameters & Group 1 & Group 2 & P value \\
\hline RDW $(\%)$ & $15.5 \pm 1.9$ & $17.7 \pm 2.7$ & $\mathrm{p}=0.02$ \\
MCV $(/ \mathrm{fL})$ & $86.7 \pm 4.9$ & $85.2 \pm 4.8$ & $\mathrm{p}>0.05$ \\
PLT $(/ \mu 1)$ & $278.9 \pm 59.6$ & $287.7 \pm 78.4$ & $\mathrm{p}>0.05$ \\
HGB $(\mathrm{gr} / \mathrm{dl})$ & $13.9 \pm 1.1$ & $13.5 \pm 1.1$ & $\mathrm{p}>0.05$ \\
\hline
\end{tabular}

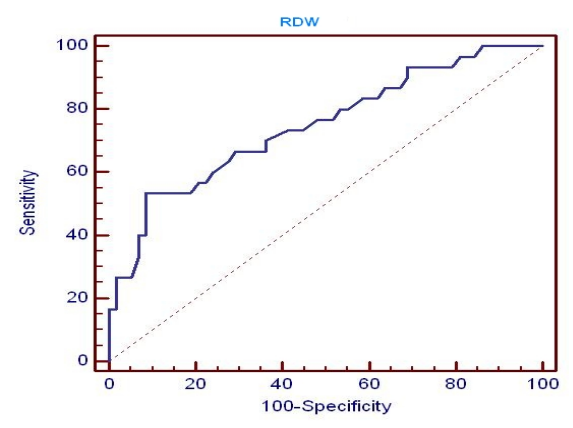

Figure 2. Receiver Operating Characteristics (ROC) Curve Analysis for RDW (AUC=0.747; p<0.001) age average of group 2 was $60,8 \pm 8,4$. There was no statistically significant difference in terms of age and sex between the groups. Mean RDW value was $15,5 \pm 1,9$ in the polyp group while it was $17,7 \pm 2,7$ in the cancer group (Figure 1). RDW value significantly increased in the cancer group when compared to the polyp group $(\mathrm{p}<0,05)$. No significant difference was found between the groups in terms of MCV, Plt and Haemoglobin (Table 1). As a result of the ROC curve analysis, it was seen that optimum RDW value for colon cancer (cut-off value) was 53,3\% sensitive and $91,4 \%$ specificity $17,5 \%$ (Area under curve $(\mathrm{AUC})=0.747 ; \mathrm{p}<0,001)$ (Figure 2).

\section{Discussion}

Colorectal cancers are still commonly seen today and they lead to high amount of mortality and morbidity. It is seen that this rate can be reduced through routine scanning and extracted guidelines (Winaver et al., 2003; Rex et al., 2000). However, these rates are still not in the desired level.

Colorectal cancers particularly have a tendency for haemorrhage more than normal and this reduces iron storages and leads to iron deficiency anaemia. And we, in this study, researched the change in RDW which is a cheaper and easier method because it is found in the standard blood count without evaluating iron storages in the patients before anaemia develops.

RDW is one of the parameters which are measured in the complete blood count and it is an indicator of the anisocytosis in the erythrocytary which circulates in the blood. Recently it was shown that RDW increased in the diseases such as rheumatoid arthritis and inflammatory intestinal disease in which cardiovascular morbidity and mortality increased, chronic and active inflammation increased (Günebakmaz et al.,2012; Nishizaki et al.,2012; Karabulut and Uzunlar, 2012; Yesil et al., 2011; Albayrak et al., 2014). RDW is used as the early indicator of increased oxidative stress, disorder in iron mobilization and iron deficiency anaemia and many studies have shown that that it increased in correlation with the inflammation markers such as CRP, IL-6 TNF a (Karabulut and Uzunlar et al., Agarval et al., 2012). The 3,845-person wide-scale cohort study by Lippi et.al also showed that RDW increased in inflammation in correlation with sedimentation and CRP.

Although there are great number studies which show that RDW increased in impaired cardiometabolic function and active inflammation, there are limited studies with solid malignity which triggers inflammation. Only two studies showed that RDW could be used as an additional parameter in the early detection of anaemia in Colorectal cancers (Spell et al., 2004; Speights et al., 1992). Moreover, Beyazit et.al. (2012) reported that increased RDW value can be a useful marker with $14,8 \%$ cut-off value and $72 \%$ sensitivity and $69 \%$ specificity in the distinguishment of malign and benign reasons of which lead to biliary obstruction. Likewise, Seretis et.al, who compared benign and malign lesions in the breast, showed that RDW increased in malign lesions but stated that it would be wrong to give a cut-off value due to the fewness of number. We, in our study, found the cut-off value as 
$53,3 \%$ and sensitivity as $91,4 \%$ and $17,5 \%$ specificity as a result of the ROC analysis.

Colorectal cancers should also be suspected in the RDW highness before anaemia develops. In our study, RDW values were higher in the cancer group when compared to the other group even after excluding the patients with anaemia. However, haemoglobin values were similar in both groups. Likewise, Baicus et.al.(2011) reported in their study that $\mathrm{MCV}$ and Platelet values were not associated with cancer. And our study also supported this.

Colorectal cancers are still commonly seen today and they lead to high amount of mortality and morbidity. It is seen that this rate can be reduced through routine scanning and extracted guidelines (Winaver et al., 2003; Rex et al., 2000). However, these rates are still not in the desired level.

Colorectal cancers particularly have a tendency for haemorrhage more than normal and this reduces iron storages and leads to iron deficiency anaemia. And we, in this study, researched the change in RDW which is a cheaper and easier method because it is found in the standard blood count without evaluating iron storages in the patients before anaemia develops.

RDW is one of the parameters which are measured in the complete blood count and it is an indicator of the anisocytosis in the erythrocytary which circulates in the blood. Recently it was shown that RDW increased in the diseases such as rheumatoid arthritis and inflammatory intestinal disease in which cardiovascular morbidity and mortality increased, chronic and active inflammation increased (Yesil et al., 2011; Gunebakmaz et al., 2012; Karabulut and Uzunlar, 2012; Nishizaki et al., 2012; Albayrak et al., 2014). RDW is used as the early indicator of increased oxidative stress, disorder in iron mobilization and iron deficiency anaemia and many studies have shown that that it increased in correlation with the inflammation markers such as CRP, IL-6 TNF a (Karabulut and Uzunlar et al., Agarval et al., 2012). The 3,845-person wide-scale cohort study by Lippi et.al also showed that RDW increased in inflammation in correlation with sedimentation and CRP.

Although there are great number studies which show that RDW increased in impaired cardiometabolic function and active inflammation, there are limited studies with solid malignity which triggers inflammation. Only two studies showed that RDW could be used as an additional parameter in the early detection of anaemia in Colorectal cancers (Speights et al., 1992; Spell et al., 2004). Moreover, Beyazit et.al. (2012) reported that increased RDW value can be a useful marker with $14,8 \%$ cut-off value and $72 \%$ sensitivity and $69 \%$ specificity in the distinguishment of malign and benign reasons of which lead to biliary obstruction. Likewise, Seretis et.al, who compared benign and malign lesions in the breast, showed that RDW increased in malign lesions but stated that it would be wrong to give a cut-off value due to the fewness of number. We, in our study, found the cut-off value as $53,3 \%$ and sensitivity as $91,4 \%$ and $17,5 \%$ specificity as a result of the ROC analysis.

Colorectal cancers should also be suspected in the
RDW highness before anaemia develops. In our study, RDW values were higher in the cancer group when compared to the other group even after excluding the patients with anaemia. However, haemoglobin values were similar in both groups. Likewise, Baicus et.al. (2011) reported in their study that MCV and Platelet values were not associated with cancer. And our study also supported this.

In conclusion, RDW attracts attention as a parameter which increases in colorectal cancers before the generation of anaemia. RDW is found in the standard complete blood count, it does not bring any extra costs and it can be easily evaluated, which strengthens the fact that it can used as a biomarker in the early detection of cancer. Nevertheless, there is a need for prospective studies which contain a higher number of patients.

\section{References}

Agarval S (2012). Red cell distribution width, inflammatory markers and cardiorespiratory fitness: Results from the National Health and Nutrition Examination Survey. Indian Heart J, 64, 380-7.

Albayrak S, Zengin K, Tanik S, et al (2014). Red cell distribution width as a predictor of prostate cancer progression. Asian Pac J Cancer Prev, 15, 7781-4.

Baicus C, Caraiola S, Rimbas M, et al (2011). Utility of routine hematological and inflammation parameters for the diagnosis of cancer in involuntary weight loss. J Investig Med, 59, 951-5.

Beyazit Y, Kekilli M, Ibis M, et al (2012). Can red cell distribution width help to discriminate benign from malignant biliary obstruc $\neg$ tion? A retrospective single center analysis. Hepatogastroenterology, 59, 1469-73.

de Gonzalo-Calvo D, de Luxan-Delgado B, Rodriguez- Gonzalez $\mathrm{S}$, et al (2012). Interleukin 6, soluble tumor necrosis factor receptor I and red blood cell distribution width as biological markers of functional dependence in an elderly population: a translational approach. Cytokine, 58, 193-8.

Gunebakmaz O, Kaya MG, Duran M, et al (2012). Red Blood Cell Distribution Width in 'Non-Dippers' versus 'Dippers'. Cardiology, 123, 154-9.

Karabulut A, Uzunlar B (2012). Correlation between red cell distribution width and coronary ectasia in the acute myocardial infarction. Clin Appl Thromb Hemost, 18, 551-2.

Lippi G, Targher G, Montagnana M, et al (2009). Relation between red blood cell distribution width and inflammatory biomarkers in a large cohort of unselected outpatients. Arch Pathol Lab Med, 133, 628-32.

Nishizaki Y, Yamagami S, Suzuki H, et al (2012). Red blood cell distribution width as an effective tool for detecting fatal heart failure in super-elderly patients. Intern Med, 51, 2271-76.

Ozkalemkas F, Ali R, Ozkocaman V, et al (2005). The bone marrow aspirate and biopsy in the diagnosis of unsuspected nonhematologic malignancy: a clinical study of 19 cases. BMC Cancer, 5, 144.

Patel KV, Semba RD, Ferrucci L, et al (2009). Red cell distribution width and mortality in older adults: a metaanalysis. J Gerontol A Biol Sci Med Sci, 65, 258-65

Rex DK, Johnson DA, Lieberman DA, et al (2000). Colorectal prevention 2000: screening recommendations of the American college of gastroenterology. Am J Gastroenterol, 95, 868-77.

Rhodes CJ, Howard LS, Busbridge M, et al (2011). Iron deficiency and raised hepcidin in idiopathic pulmonary 
Serden Ay et al

arterial hypertension: clinical prevalence, outcomes, and mechanistic insights. J Am Coll Cardiol, 58, 300-9.

Seretis C, Seretis F, Lagoudianakis E, et al (2013). Is red cell distribution width a novel biomarker of breast cancer activity? data from a pilot study. J Clin Med Res, 5, 121-6.

Speights VO, Johnson MW, Stoltenberg PH, et al (1992). Complete blood count indices in colorectal carcinoma. Arch Pathol Lab Med, 116, 258-60.

Spell DW, Jones DV, Jr., Harper WF, et al (2004). The value of a complete blood count in predicting cancer of the colon. Cancer Detect Prev, 28, 37-42.

Winawer S, Fletcher R, Rex D, et al (2003). Colorectal cancer screening and surveillance: clinical guidelines and rationaleupdate based on new evidence. Gastroenterology, 124, 544-60.

Yesil A, Senates E, Bayoglu IV, et al (2011). Red cell distribution width: a novel marker of activity in inflammatory bowel disease. Gut Liver, 5, 460-7. 